\title{
EGF signaling activates proliferation and blocks apoptosis of mouse and human intestinal stem/progenitor cells in long-term monolayer cell culture
}

\author{
Atsushi Suzuki ${ }^{1,2}$, Sayaka Sekiya ${ }^{1,2}$, Eriko Gunshima ${ }^{2}$, Setsuko Fujii ${ }^{1}$ and Hideki Taniguchi ${ }^{1,3}$
}

The homeostatic renewal of the intestinal epithelium depends on regulation of proliferation and differentiation of stem/ progenitor cells residing in a specific site, called the 'stem cell niche.' Thus, the reconstitution of the microenvironment of the stem cell niche may allow us to maintain intestinal stem/progenitor cells in culture for a longer period. Although epidermal growth factor (EGF) is conventionally used as a supplement of intestinal epithelial cell culture, little has been known regarding a role of EGF signaling in a stem/progenitor cell population. In this study, we attempted to clarify the role of EGF signaling in intestinal stem/progenitor cells, and to establish a culture system in which these cells could be maintained with normal differentiation potential. We first examined the expression pattern of EGF and its receptor, EGFR, and inhibited EGF signaling in mouse intestines. Next, we cultured intestinal cells isolated from mouse and human intestines in the presence of EGF and analyzed the function of EGF signaling in cultured cells. In both embryonic and adult mouse intestines, EGFR and EGF were expressed in immature epithelial cells and adjacent fibroblasts, respectively, and EGF signaling was essential to activate proliferation and inhibit apoptosis of intestinal stem/progenitor cells. Activation of EGF signaling also stimulated proliferation and suppressed apoptosis, both of which are necessary to maintain mouse and human intestinal epithelial cells in culture. Moreover, in these cultured epithelial cells, putative intestinal stem/progenitor cells persisted longer, and gave rise to different types of differentiated intestinal epithelial cells. We conclude that EGF signaling is indispensable for activation of proliferation and inhibition of unexpected cell death, not only in the intestinal stem cell niche, but also in culture of primitive intestinal epithelial cells.

Laboratory Investigation (2010) 90, 1425-1436; doi:10.1038/labinvest.2010.150; published online 16 August 2010

KEYWORDS: cell culture; epidermal growth factor; intestine; stem/progenitor cells

The intestinal epithelium initially develops as a multistratified cell layer of the endoderm, which subsequently converts into an epithelial monolayer and finally creates a characteristic architecture composed of villi and crypts. ${ }^{1}$ Villi extend into the intestinal lumen, and consist of non-proliferative, terminally differentiated epithelial cells, for example, enterocytes, goblet cells, and enteroendocrine cells. In addition to these cells, differentiated Paneth cells are located at the crypt base. These four types of epithelial cells coordinately operate in maintaining intestinal function, but disappear from the epithelium within a week by apoptosis. Therefore, to provide a continuous source of differentiated cells, the intestinal epithelium renews rapidly through vigorous proliferation of intestinal stem cells (ISCs) and progenitor cells residing in the crypts. This balance of proliferation and apoptosis is important for the maintenance of intestinal tissue homeostasis.

A specific microenvironment, called the 'stem cell niche,' is required for self-renewing cell divisions of undifferentiated cells and a continuous supply of differentiated cells. ${ }^{2}$ The function of the niche in the intestinal crypt requires the presence of extracellular matrix (ECM) and humoral factors derived from fibroblasts in the mesenchymal tissue surrounding the epithelium and epithelial cells proliferating

\footnotetext{
${ }^{1}$ Research Unit for Organ Regeneration, Center for Developmental Biology, Chuo-ku, Kobe, Hyogo, Japan; ${ }^{2}$ Division of Organogenesis and Regeneration, Post-Genome Science Center, Medical Institute of Bioregulation, Kyushu University, Higashi-ku, Fukuoka, Fukuoka, Japan and ${ }^{3}$ Department of Regenerative Medicine, Graduate School of Medicine, Yokohama City University, Yokohama, Kanagawa, Japan

Correspondence: H Taniguchi, MD, PhD, Department of Regenerative Medicine, Graduate School of Medicine, Yokohama City University, 3-9 Fuku-ura, Kanazawa-ku, Yokohama, Kanagawa 236-0004, Japan. E-mail: rtanigu@med.yokohama-cu.ac.jp

Received 2 January 2010; revised 8 June 2010; accepted 1 July 2010
} 
within the crypt. ${ }^{3-6}$ Both undifferentiated and differentiated epithelial cells in the intestine adhere to the basement membrane, which is composed of various ECM components, by many types of integrins. ${ }^{5,6}$ Thus, variation of integrins and ECM components in the villus-crypt axis may be related to distinctive features of the crypt niche. Signals mediated by humoral factors, including Wnt, Ephrins, and bone morphogenetic protein (BMP), and their receptors are also essential in order to regulate the fate of different cell lineages in the intestine. ${ }^{3}$ The canonical Wnt pathway activates proliferation of immature cells in the crypt and maturation of Paneth cells. ${ }^{7,8}$ Interaction between Ephrins and their tyrosine kinase receptors, Eph, whose expression is regulated through Wnt pathway, controls cell migration patterns within the crypt and segregates cells along the villus-crypt axis. ${ }^{9,10}$ BMP signaling negatively regulates proliferation of undifferentiated cells in the crypt through suppression of the Wnt signaling pathway. ${ }^{11,12}$ However, the expression of Noggin, an inhibitor of BMP signaling, in epithelial cells and adjacent fibroblasts within the crypt allows proliferation of ISCs and progenitor cells. ${ }^{12}$ Moreover, growth factor receptor signaling that initiates $\operatorname{PtdIns}(3,4,5)$ kinase (PI3K) pathway induces phosphorylation of Akt and activates proliferation of immature cells in the crypt, a process that is strictly regulated by the function of phosphatase and tensin homologue (PTEN). ${ }^{12,13}$ The lack of PTEN results in de novo crypt formation because of an excess of ISCs, leading to initiation of intestinal polyposis. ${ }^{13}$ Akt induces cell survival and cell cycle progression through phosphorylation of multiple target proteins, including $\beta$-catenin, an effector of canonical Wnt signaling. ${ }^{13}$ Therefore, the cross-talk among $\mathrm{Wnt} / \beta$-catenin, $\mathrm{BMP}$, and PTEN/PI3K/Akt signaling is critical for the control of intestinal tissue homeostasis. In fact, in humans, mutations in adenomatosis polyposis coli (APC: a negative regulator of Wnt signaling), BMPR1A (a BMP receptor), Smad4 (a transducer of BMP signaling), and PTEN are responsible for familial adenomatous polyposis (FAP), juvenile polyposis syndrome, juvenile intestinal polyposis, and Cowden disease, respectively. ${ }^{14-17}$ On the other hand, interactions between immature epithelial cells in the crypt, mediated by the Notch signaling pathway, are also important for determination of cell fate in the intestine. ${ }^{18,19}$

A method for long-term intestinal epithelial cell culture has been developed to establish an in vitro model for the culture of ISCs and progenitor cells. ${ }^{20-22}$ The establishment of cell culture system that would allow the maintenance of viable intestinal cells for a longer period requires a microenvironment that mimics the stem cell niche in the crypt of the intestine. Co-culture of intestinal epithelial cells with fibroblasts reproduces the interaction between these two types of cells via basement membrane components and the supply of humoral factors, which mediate cell survival and proliferation, eventually leading to the maintenance and the differentiation of epithelial cells in culture. ${ }^{23-24}$ In the presence of ECM components and/or humoral factors responsible for in vivo crypt cell proliferation and survival, undifferentiated intestinal epithelial cells could be maintained in culture in a proliferative state and give rise to differentiated epithelial cells, even without supporting cells such as fibroblasts.

Accordingly, we attempted to determine a humoral factor capable of inducing proliferation and supporting viability of immature cells residing in the developing epithelia and crypts of intestines. A previous study demonstrated that the suppression of epidermal growth factor (EGF) signaling in culture of embryonic intestinal tissues inhibited proliferation of epithelial cells and increased the number of apoptotic cells in the epithelia. ${ }^{25}$ Furthermore, in a mouse model of human FAP, an inhibitor of the EGF receptor (EGFR) kinase attenuated polyp formation. ${ }^{26}$ Thus, EGF could be considered as a possible candidate for the humoral factor that allows maintenance of viable immature crypt cells in vitro, as well as in vivo. Indeed, EGF has been conventionally used as a supplement in culture of intestinal epithelial cells, while little has been known about a role of EGF signaling in an intestinal stem/progenitor cell population. In this study, we examined and clarified the role of EGF in intestinal stem/progenitor cells and used it to achieve a prolonged monolayer culture of these cells. Normal intestinal stem/progenitor cells maintained in culture could be used not only to study digestive diseases, but also to screen for the pharmacological effects of new drugs.

\section{MATERIALS AND METHODS Cell Culture}

Cell suspensions of intestinal tissue were prepared from CD-1 E14.5 fetal mice and 9-week-old adult mice. The tissues were carefully dissected under the microscope and minced with a surgical blade. Small pieces of the tissue were then placed in Hanks' balanced salt solution (GIBCO BRL) containing $1 \mathrm{mmol} / \mathrm{l} \mathrm{EGTA}$, and incubated for $10 \mathrm{~min}$ at $37^{\circ} \mathrm{C}$. After washing with PBS, tissue fragments were digested by incubating in Hanks' balanced salt solution containing $5 \mathrm{mmol} / \mathrm{l}$ $\mathrm{CaCl}_{2}$ (pH 7.4) and $1 \mathrm{mg} / \mathrm{ml}$ collagenase (Sigma) for $15-20 \mathrm{~min}$ at $37^{\circ} \mathrm{C}$. Following exclusion of residual tissues by filtration, triturated cells were washed in our standard culture medium $(\mathrm{SCM})^{27}$ before beginning cell culture. SCM is a 1:1 mixture of Dulbecco's modified Eagle medium and F-12, supplemented with $10 \%$ fetal bovine serum (FBS), insulin $(1 \mu \mathrm{g} / \mathrm{ml})$, dexamethasone (Dex) $\left(1 \times 10^{-7} \mathrm{~mol} / \mathrm{l}\right)$, nicotinamide (NAM) $(10 \mathrm{mmol} / \mathrm{l})$, L-glutamine $(2 \mathrm{mmol} / \mathrm{l})$, $\beta$-mercaptoethanol $(50 \mu \mathrm{mol} / \mathrm{l})$, penicillin/streptomycin, and EGF $(20 \mathrm{ng} / \mathrm{ml})$ (Sigma). Isolated E14.5 embryonic intestinal cells were placed in non-adhesive, round-bottom wells of 96-well plates (Nunc, Cat No: 145399) at concentrations of $2-4 \times 10^{5}$ cells per well. Aggregates formed by intestinal cells were then transferred into Petri dishes $(6 \mathrm{~cm}$ in diameter; $\mathrm{BD}$ Biosciences) and allowed to adhere to the bottom of dishes in the presence of EGF. For adult mouse intestinal cells, $1-5 \times 10^{6}$ cells were directly plated into Petri dishes 
$(6 \mathrm{~cm}$ in diameter). Epithelial cell expansion could be observed when embryonic or adult mouse intestinal cells were cultured for $>3$ or 8 months, respectively. If fibroblasts still existed and proliferate in culture, cultured cells should be treated with mono-iodoacetate $(4 \mu \mathrm{g} / \mathrm{ml})$ for $3-5 \mathrm{~h}$. Epithelial cells were finally transferred to tissue-culture dishes to be maintained in culture for a longer period and analyzed. For culture of adult human intestinal cells, we obtained post-isolated cells from Dainippon Sumitomo Pharma, Japan (Cat No.CS-ABI-519) and cultured them in attached CS-2.0 serum-free medium or SCM in the presence of EGF.

\section{Gene Expression Analysis}

Reverse-transcription polymerase chain reaction (RT-PCR) was conducted as described. ${ }^{28-29}$ Information regarding PCR primers is presented in Supplementary Tables 1 and 2 and in our previous papers. ${ }^{27,29,30}$

\section{Immunostaining}

Frozen sections of intestinal tissues were fixed by $4 \%$ paraformaldehyde for $5 \mathrm{~min}$ at $4{ }^{\circ} \mathrm{C}$. After washing in PBS and blocking, tissue sections were incubated with primary antibodies against EGFR (Santa Cruz), EGF (Chemicon), E-cadherin (E-cad) (BD Biosciences), BrdU (Amersham), and cleaved caspase-3 (Cell signaling). Paraffin sections were deparaffinized and rehydrated, and antigen retrieval was performed by microwaving slides in $0.01 \mathrm{M}$ citrate buffer (pH 6). After washing and blocking, these paraffin sections were incubated with an anti-BrdU antibody. Cultured cells were fixed as described previously ${ }^{29}$ and incubated with primary antibodies against E-cad, Zo-1 (Zymed), Villin (Santa Cruz), Musashi-1 (Chemicon), serotonin (Immunostar), chromogranin A (Santa Cruz), gastrin (Santa Cruz), secretin (Chemicon), somatostatin (Affiniti Research Products), gastric inhibitory polypeptide (GIP) (Chemicon), synaptophysin (Dako), lysozyme (Dako), Ki-67 (Abcam), and $\beta$-galactosidase (Promega). After washing, paraffin sections were incubated with a horseradish peroxidaseconjugated secondary antibody (1:500; Dako), and frozen sections and cultured cells were incubated with Alexa 488and/or Alexa 555-conjugated secondary antibodies (1:200; Molecular Probes) with DAPI. After final washing, stained tissues and cells were viewed using an Olympus fluorescence microscope or a Zeiss LSM510 laser scanning microscope.

\section{Analysis of Cell Proliferation and Apoptosis}

Cells were cultured in the presence or absence of inhibitors of the EGF signaling pathway, such as AG1478 $(1 \mu \mathrm{M})$ (Wako), PD98059 $(100 \mu \mathrm{M})$ (Calbiochem), or LY294002 $(100 \mu \mathrm{M})$ (Calbiochem), for 1 day before analysis. Apoptotic cells stained with allophycocyanin (APC)-conjugated Annexin V (BD Biosciences) and BrdU-incorporated cells stained using APC BrdU Flow Kit (BD Biosciences) were analyzed with FACS Aria (BD Biosciences).

\section{Western Blotting}

Antibodies against EGFR (1:1000; Cell Signaling), phosphorylated (p) EGFR (1:1000; Cell Signaling), MEK1/2 (1:1000; Cell Signaling), pMEK1/2 (1:1000; Cell Signaling), ERK1/2 (1:1000; BD Biosciences), pERK1/2 (1:1000; BD Biosciences), Akt (1:250; BD Biosciences), pAkt (1:250; BD Biosciences), and $\beta$-actin (1:2000; Abcam) were used for western blotting analysis. Interaction of Ras and the Ras-binding domain of Raf-1 was analyzed using the Ras Activation Assay Kit (Upstate Biotechnology).

\section{RESULTS}

\section{Expression Pattern of EGF/EGFR and the Role of EGF Signaling in Developing and Adult Mouse Intestines}

We examined the expression pattern of EGF and EGFR in the developing and adult mouse intestines by co-immunofluorescence staining. In the developing intestine, EGFR is expressed in epithelial cells that are marked by the expression of E-cad, whereas EGF expression is observed in the mesenchymal tissue surrounding the epithelium (Figure 1a). The expression of EGFR is restricted into epithelial cells within the crypt of adult mouse intestine, but EGF is still expressed in only mesenchymal cells (Figure 1b). To determine the role of EGF signaling in the developing and adult mouse intestine, we introduced AG1478, an inhibitor of EGFR kinase, into pregnant or non-pregnant adult mice. Inhibition of EGF signaling resulted in a decrease in proliferating cells in the intestinal epithelia of mouse embryos and the crypts of adult mouse intestines (Figures $1 \mathrm{c}$ and d). Moreover, in the presence of AG1478, the number of apoptotic cells increased in the epithelia of developing mouse intestines and an upper region of the crypt of adult mouse intestines (Figure 1d). These data indicate that proliferation and survival of immature intestinal epithelial cells that reside in the developing mouse intestine and the crypt of adult mouse intestine depend on the activation of EGF signaling. This signaling is mediated by an association between EGFR and EGF, which are expressed in epithelial cells and mesenchymal cells in the intestine, respectively.

\section{Culture of Intestinal Epithelial Cells Isolated from Developing and Adult Mouse Intestines with EGF}

To examine whether EGF can maintain intestinal epithelial cells in culture, we cultured intestinal cells isolated from E14.5 mouse embryos as a simple monolayer in the presence of EGF. The morphologically identified epithelial cells were found after the initiation of culture, but these cells disappeared within 3 weeks and fibroblasts substitutively grew to cover the surface of culture dishes (Figure 2a). We next deposited embryonic intestinal cells into non-adhesive, round-bottom wells of 96-well plates to allow cells to form aggregates (Figure $2 \mathrm{~b}$ ). Under these culture conditions, we expected to induce aggregation of epithelial cells through E-cad homophilic binding, thereby decreasing the number of fibroblasts in culture and enriching for epithelial cells. 

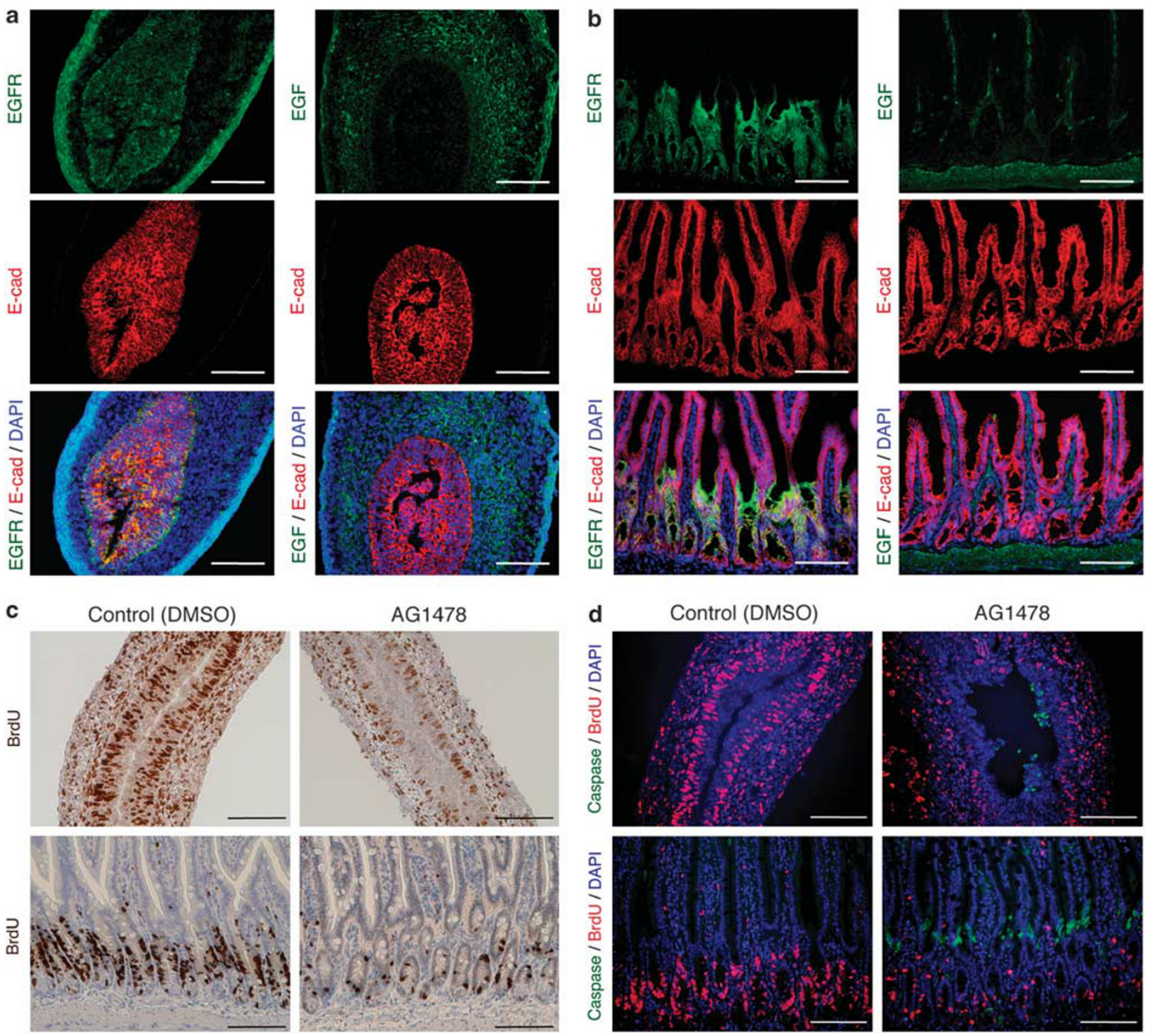

AG1478
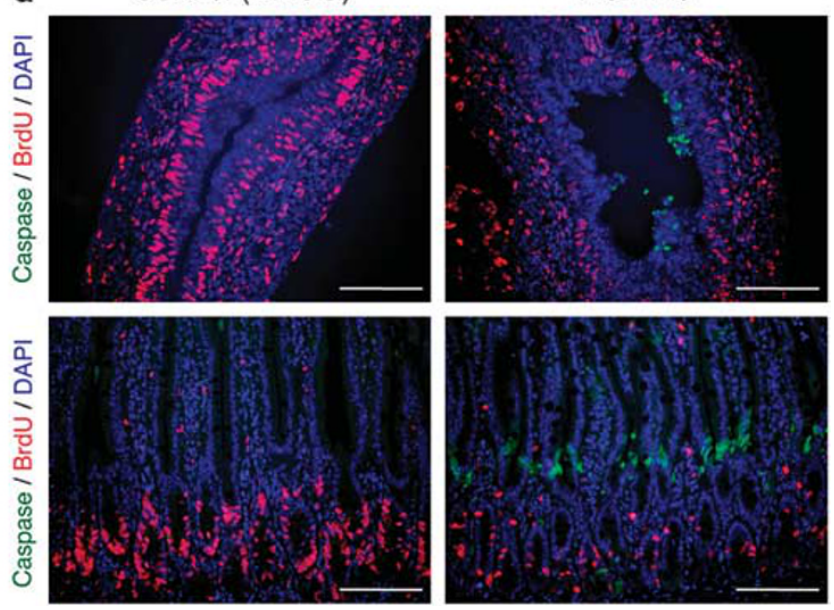

Figure 1 Expression of EGF/EGFR and the role of EGF signaling in mouse intestines. (a, b) Co-immunofluorescence staining of E-cad and EGFR (left panels) or EGF (right panels) for E14.5 developing (a) and 9-week-old adult mice intestines (b). DNA is stained with DAPI. (c) Immunohistochemical staining of BrdU in E14.5/developing (upper panels) and adult (lower panels) mouse intestine, counter-stained with eosin, following intraperitoneal injection of DMSO (control) or AG1478 (3 mg per mouse) twice at intervals of $15 \mathrm{~h}$. (d) Co-immunofluorescence staining of BrdU and cleaved caspase-3 for E14.5/developing (upper panels) and adult (lower panels) mouse intestines after injection of DMSO or AG1478. DNA is stained with DAPI. Scale bar, $100 \mu \mathrm{m}$.

As expected, various sizes of aggregates were found in culture, and an epithelial sheet-like structure was observed in each aggregate (Figures $2 \mathrm{c}$ and $\mathrm{d}$ ). After $5-7$ days of culture, floating aggregates were transferred into Petri dishes and allowed to adhere. Then, cells within aggregates dispersed nearby and proliferated in the presence of EGF (Figure 2e). These scattered cells seemed like epithelial cells that adhered tightly to other cells and continued proliferating as a monolayer. In conditions wherein we observed extensive growth of epithelial-like cells, fibroblasts became senescent and gradually disappeared from the culture. As a consequence of this protocol, epithelial-like cells that were originally derived from developing mouse intestines could be maintained in culture for $>6$ months and be cryopreserved in the liquid nitrogen (Figure 2f). We provisionally called these cells fetal mousederived intestinal progenitor (FIP) cells. Moreover, in addition to FIP cells, we established a monolayer culture of adult mouse-derived intestinal progenitor (AIP) cells in the presence of EGF (Figures $2 \mathrm{~g}$ and $\mathrm{h}$ ). In the case of AIP cells, however, cell aggregation was not required for the proliferation of epithelial-like cells, though the rate of establishment of AIP cell cultures was much lower than that of FIP cell 

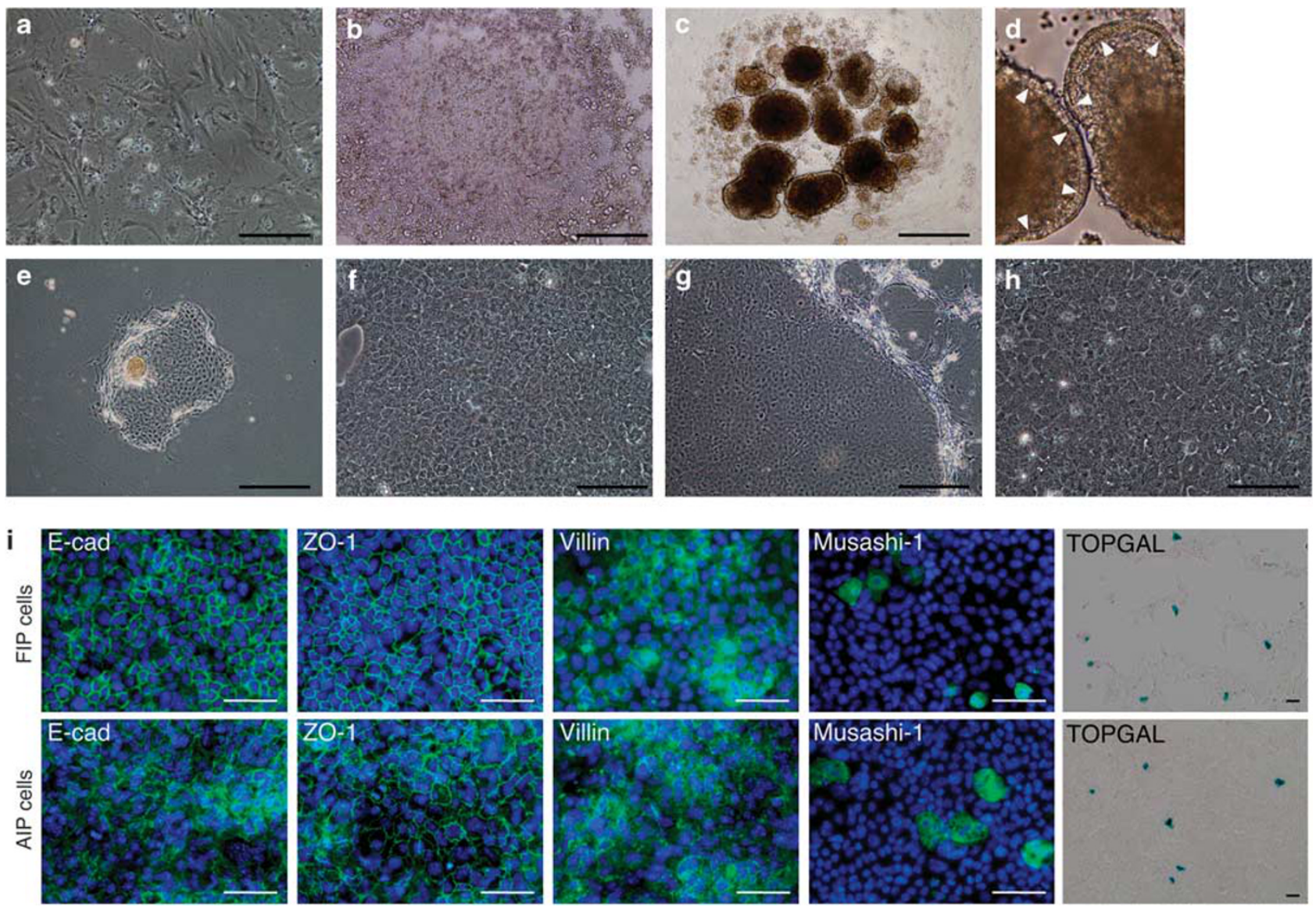

Figure 2 Establishment of mouse FIP/AIP cell cultures (a) Fibroblasts remained in a monolayer culture of E14.5 mouse intestinal cells, while epithelial cells completely disappeared from culture within 3 weeks. (b) For cell-aggregate formation, E14.5 mouse intestinal cells were deposited into non-adhesive, round-bottom wells of 96-well plates. (c) Two days after the initiation of culture, various sizes of aggregates were formed. (d) A magnified picture of aggregates shown in c. Arrowheads denote epithelial sheet-like structures in aggregates. (e) Expansion of epithelial-like cells from aggregates after transferring aggregates into Petri dishes. (f) A representative picture of mouse FIP cells maintained in culture. (g) Expansion of epithelial-like cells in culture of adult mouse-derived intestinal cells. (h) A representative picture of mouse AIP cells maintained in culture. (i) Immunofluorescence staining of E-cad, Zo-1, Villin, and Musashi-1 for mouse FIP/AIP cells cultured in the presence of EGF. DNA is stained with DAPI. X-gal staining was also conducted for mouse FIP/AIP cells transfected with the TOP-GAL construct. Scale bar, $50 \mu \mathrm{m}(\mathbf{i}), 100 \mu \mathrm{m}(\mathbf{a}, \mathbf{f}, \mathbf{h})$, and $500 \mu \mathrm{m}(\mathbf{b}, \mathbf{c}, \mathbf{e}, \mathbf{g})$.

cultures. Immunofluorescence analysis showed that both FIP and AIP cells expressed E-cad and Zo-1, which are markers for epithelial cells, as well as Villin, an actin-binding protein expressed in intestinal epithelial cells (Figure 2i). In cultures of these cells, cells expressing Musashi-1, a recently identified marker of ISCs and progenitor cells were also included, and several genes expressed in progenitor cells, such as Math-1 and neurogenin-3, were detected with RT-PCR (Figure $2 \mathrm{i}$ and data not shown). RT-PCR analysis also showed the expression of leucine-rich-repeat-containing G-protein-coupled receptor 5 (Lgr5), which is a novel marker of ISCs, ${ }^{31}$ in culture of these cells (Supplementary Figure 1). Moreover, transient transfection of TOP-GAL constructs (a generous gift from E Fuchs) into FIP and AIP cells revealed cells with lacZ activity (Figure $2 \mathrm{i}$ ). The majority of these lacZ ${ }^{+}$cells were positive for Ki-67, a marker of proliferating cells (Supplementary Figure 2). Also, the number of $\mathrm{BrdU}^{+}$proliferating cells decreased in culture of FIP and AIP cells with DKK1, an inhibitor of the Wnt signaling pathway (Supplementary Figure 3). These results suggest that ISCs and/or progenitor cells with $\beta$-catenin transcriptional activity are included in these two types of cell cultures.

\section{Differentiation of FIP and AIP Cells after Removing EGF from Culture}

We next examined the potential for differentiation in FIP and AIP cells. As shown in Figure 1, EGF signaling activates proliferation of immature cells in the developing intestinal epithelium and the crypts of adult intestine, but not that of differentiated cells within villi of adult intestine. This suggests that inactivation of EGF signaling is required for the differentiation of intestinal epithelial cells. Thus, we excluded EGF from the culture and then analyzed the expression of markers for four distinct types of differentiated intestinal epithelial 

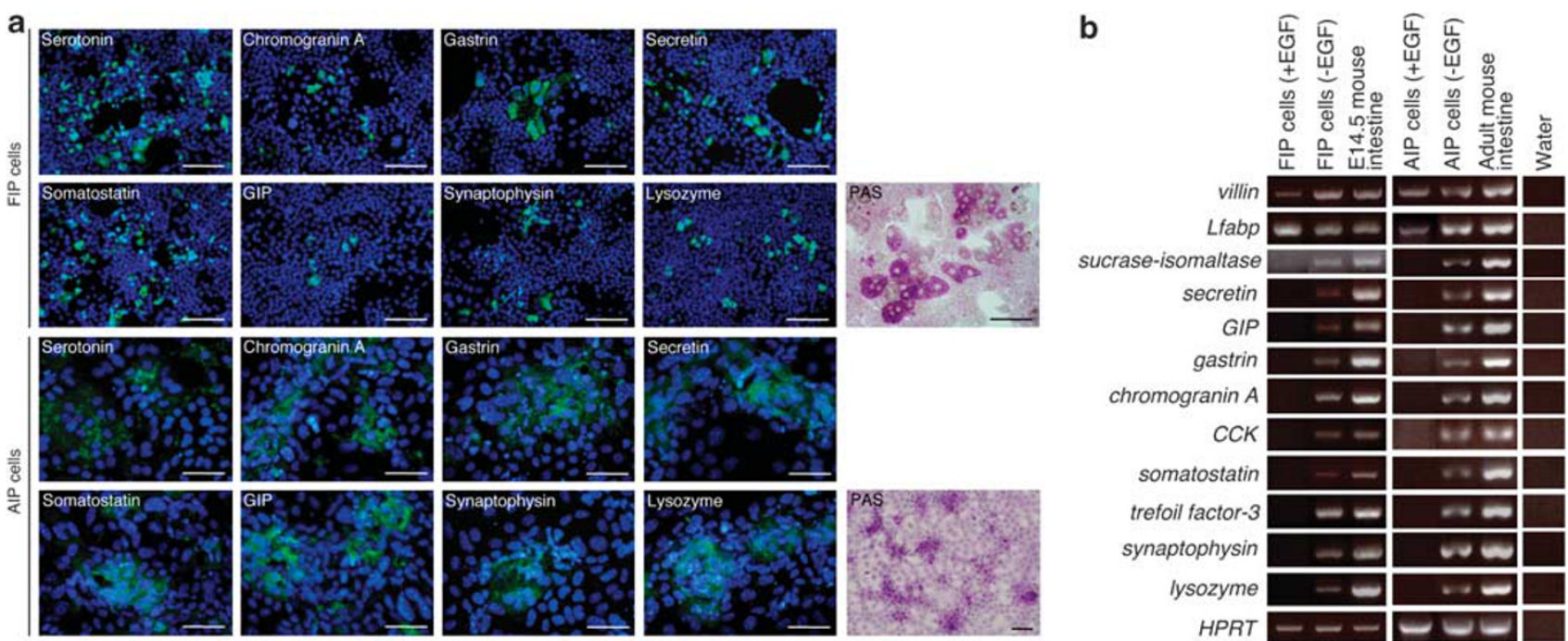

Figure 3 Differentiation of mouse FIP/AIP cells in culture. (a) Immunofluorescence staining of mouse FIP/AIP cells for serotonin, chromogranin A, gastrin, secretin, somatostatin, GIP, synaptophysin, and lysozyme, following culture for 7 days without EGF. EGF was removed from the culture medium when cells completely covered the bottom of culture dishes. DNA is stained with DAPI. PAS-positive cells also appeared in the absence of EGF (and, for AIP cells, culture medium was supplemented with DAPT). Scale bar, $100 \mu \mathrm{m}$ (FIP cells) and $50 \mu \mathrm{m}$ (AIP cells). (b) RT-PCR analysis of intestinal gene expression in mouse FIP/AIP cells. These cells were cultured with EGF throughout the culture (shown as + EGF) or in the absence of EGF for 7 days after removing EGF from culture medium (shown as -EGF). As positive or negative controls, we used cDNA prepared from E14.5 and adult mouse intestines or water, respectively. Hypoxanthine phosphoribosyltransferase (HPRT) was used as an internal control.

cells. When we examined the differentiation of FIP and AIP cells culture with EGF, it was difficult to find cells expressing markers for differentiated epithelial cells (Supplementary Figure 4 and Figure $3 \mathrm{~b}$ ). One week after the removal of EGF, however, FIP and AIP cells stopped proliferating and differentiated into cells expressing enteroendocrine cell markers serotonin, chromogranin A, gastrin, secretin, somatostatin, GIP, synaptophysin, and the Paneth cell marker lysozyme (Figure 3a); the number of apoptotic cells was also increased (see Figure 4). The goblet cells that produce mucin in the intestine are detected by mucin-staining methods, including periodic acid-Schiff (PAS). In the absence of EGF, we found PAS-positive cells within both FIP and AIP cell cultures, suggesting goblet cell differentiation (Figure $3 \mathrm{a}$ ). To induce PAS-positive cells in AIP cell cultures more frequently, we supplemented the culture medium with the Notch signaling inhibitor $\mathrm{N}$-[N-(3,5-difluorophenacetyl)-L-alanyl $]-S$ phenylglycine t-butyl ester (DAPT). It has been reported that inhibition of Notch signaling induces differentiation of goblet cells from ISCs and/or progenitor cells in adult mouse intestine. ${ }^{18,19}$ We next performed RT-PCR analysis on samples from FIP and AIP cell cultures. In addition to multiple markers for enteroendocrine cells, such as secretin, GIP, gastrin, chromogranin A, cholecystokinin (CCK), somatostatin, and synaptophysin, we also detected the enterocyte marker Lfabp and sucrase-isomaltase, the goblet cell marker trefoil factor-3, and the Paneth cell marker lysozyme (Figure 3b). These data indicate that both FIP and AIP cell cultures that contain EGF as a supplement allow maintenance and proliferation of undifferentiated cells; in the absence of EGF, however, a differentiation program is activated to generate four different types of intestinal epithelial cells.

\section{EGF Signaling Activates Proliferation and Inhibits Apoptosis of FIP and AIP Cells}

To clarify the effect of EGF signaling in FIP and AIP cell cultures, we examined proliferation and survival of these cells in culture with/without inhibitors for EGF signaling. Microscopic observation and FACS analysis revealed that, similar to the result of EGF depletion from culture medium, an extensive decrease in the number of $\mathrm{BrdU}^{+}$proliferating cells and an increase in the number of AnnexinV ${ }^{+}$apoptotic cells occurred after culture for 1 day in the presence of EGF with AG1478. Similarly, the number of $\mathrm{BrdU}^{+}$cells decreased, but the number of AnnexinV ${ }^{+}$cells increased, in culture supplemented with a mitogen-activated protein (MAP) kinase cascade inhibitor PD98059 or a PI3K inhibitor LY294002 (Figures $4 \mathrm{a}$ and $\mathrm{b}$ ). In the presence of these inhibitors, FIP and AIP cells initiated differentiation into intestinal epithelial cells, similar to the case of culture without EGF (Supplementary Figure 5). In both FIP and AIP cell cultures, EGF rapidly induced EGFR activation, as assessed by phosphorylaton of four distinct tyrosine residues, and then led to phosphorylation of the downstream signaling targets, MEK1/2, ERK1/2, and Akt (Figure 5a). Also, EGFstimulated interaction of Ras and Raf-1, which is required to activate the MAP kinase cascade, in culture of FIP and AIP cells (Figure 5b). These data demonstrate that EGF acts as an activator and a suppressor for proliferation and apoptosis, respectively, of both FIP and AIP cells. EGF acts through 
a

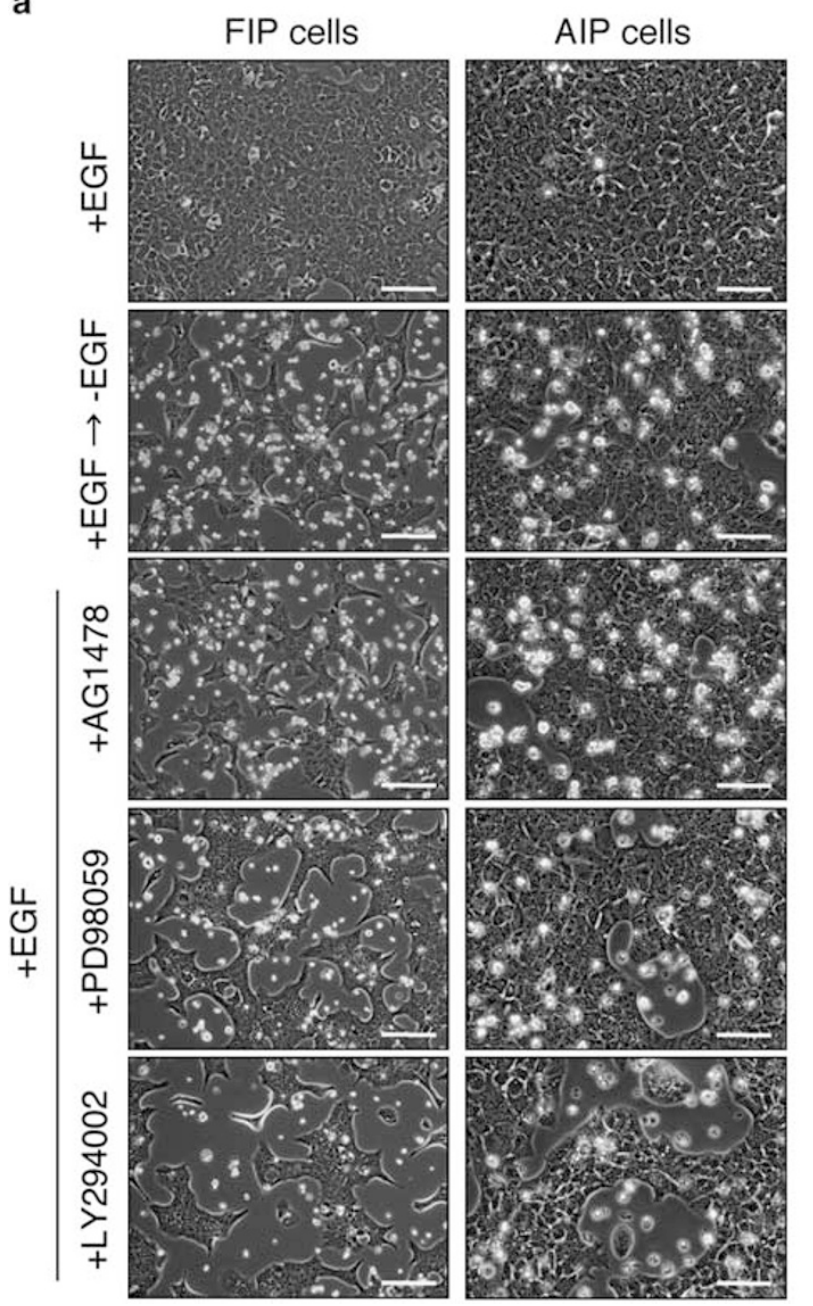

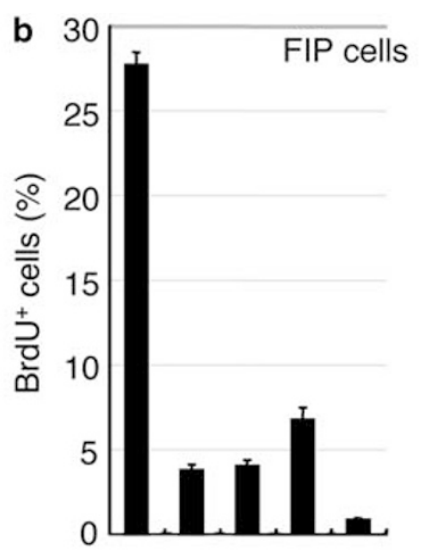

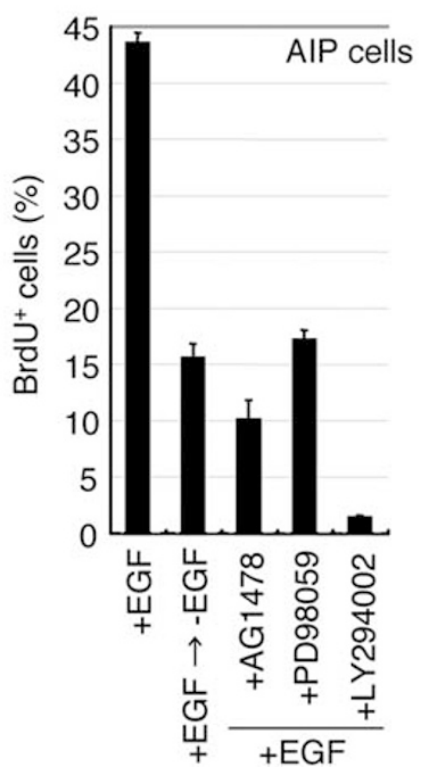

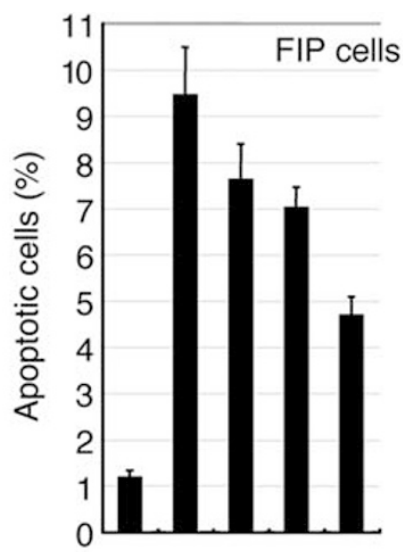

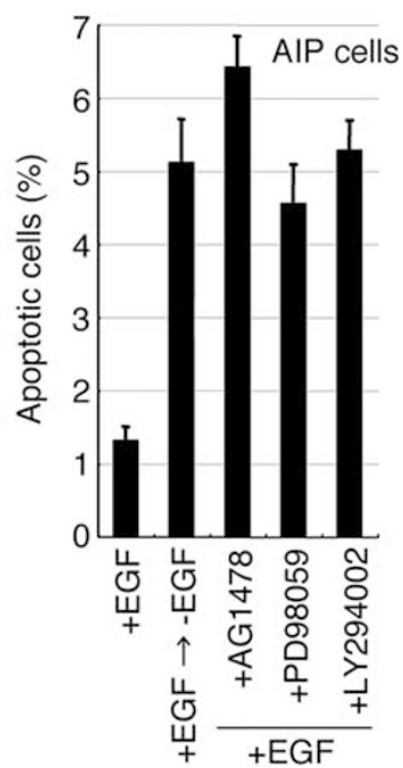

Figure 4 EGF signaling stimulates proliferation and inhibits apoptosis of mouse FIP/AIP cells. (a) Phase pictures of mouse FIP/AIP cells after 1 day of culture with or without inhibitors for EGF signaling, such as AG1478, PD98059, or LY294002, in the presence of EGF. As control experiments, these cells were also cultured with EGF throughout the culture or in the absence of EGF for 1 day after removing EGF from culture medium. We added inhibitors or removed EGF when cells occupied $70-80 \%$ of the surface of culture dishes. Scale bar, $100 \mu \mathrm{m}$. (b) The percentages of BrdU ${ }^{+}$proliferating cells (left graphs) and AnnexinV ${ }^{+}$apoptotic cells (right graphs) in the same culture conditions as shown in a were analyzed by flow cytometry.

activation of the PI3K/Akt and MAP kinase cascades in these cells.

\section{Culture of Intestinal Epithelial Cells Isolated from Adult Human Intestines with EGF}

Normal intestinal epithelial cells isolated from human intestines will be useful for testing novel therapeutic strategies targeted against intestinal disorders, as well as for screening pharmaceuticals. As an extension of the mouse study presented here, we attempted to maintain human intestinal epithelial cells in culture. Commercially available human intestinal epithelial cells proliferated in attached CS-2.0 serum-free medium with or without EGF, but could not survive after the initial passage of cells. These cells, however, could be maintained with proliferation in SCM supplemented with EGF, and cryopreserved in liquid nitrogen (Figure 6a). To determine which substances supplemented in SCM is important for the maintenance of intestinal epithelial cells, we removed each supplement from SCM, such as FBS, insulin, Dex, or NAM, and cultured intestinal epithelial cells. In the absence of FBS, proliferation of epithelial cells was almost completely blocked, while it was partially suppressed in each insulin-, Dex-, or NAM-depleted SCM (Supplementary Figure 6A). As proliferation of a type of cells sometimes depends on the lot of FBS, we next examined whether intestinal epithelial cells could only proliferate with a special FBS supplemented in SCM. As the result, no significant difference was found among 11 lots of FBS in proliferation of intestinal epithelial cells, implying that the presence of FBS in a medium is important, but any lots of FBS could be used for culture of these cells (Supplementary Figure 6B).

Similar to mouse FIP and AIP cells, human intestinal epithelial cells expressed E-cad and Zo-1, and a portion of 


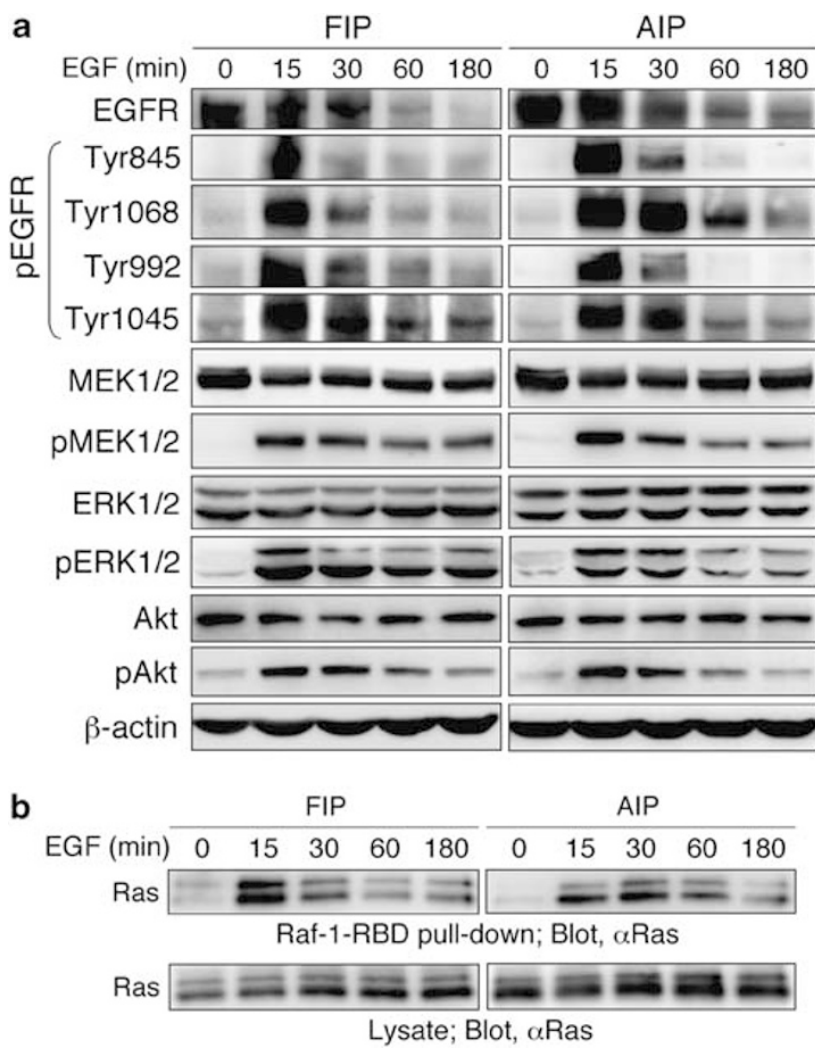

Figure 5 Activation of EGF signaling pathway in mouse FIP/AIP cells. (a) Cells were cultured in the absence of EGF for $17 \mathrm{~h}$ and then stimulated with EGF $(20 \mathrm{ng} / \mathrm{ml})$ for the indicated times at $37^{\circ} \mathrm{C}$. Cell lysates were collected from these cells and subjected to western blotting analysis. (b) After the stimulation of mouse FIP/AIP cells by EGF, interaction between activated Ras and Raf-1 was analyzed by a Raf-1-Ras-binding domain (RBD) pull-down assay, in which cell lysates were incubated with agarose bound with a glutathione S-transferase fusion protein that corresponds to the human RBD of Raf-1, followed by immunoblotting of Ras using anti-RAS antibody.

these cells were positive for Musashi- 1 and $\beta$-catenin transcriptional activity (Figure 6b). Cells marked with $\beta$-catenin transcriptional activity were also positive for $\mathrm{Ki}-67$, and, in culture with DKK1, the number of $\mathrm{BrdU}^{+}$cells decreased (Supplementary Figures 2 and 3). RT-PCR analysis also showed the expression of villin, Math-1, and neurogenin-3 in culture of these cells (data not shown). Interestingly, Lgr5 was also expressed in these cells, as well as in mouse FIP and AIP cells (Supplementary Figure 7). Thus, we decided to call these cells human AIP (hAIP) cells. Confocal microscopic Z-stack analysis of hAIP cells showed that Zo-1 and E-cad protein was localized at the upper and lower region of the lateral membrane of cultured cells, respectively, suggesting that an important cellular feature of polarized normal intestinal epithelial cells was maintained in hAIP cell culture (Figure $6 c)$. After hAIP cells completely covered the bottom of culture dishes as a monolayer sheet, we found cells that formed dome-like structures (Figures $6 \mathrm{~d}$ and e). These dome-like structures, derived from hAIP cells, resembled those from
Caco-2 cells, a well-known cell line obtained from human colon carcinoma, ${ }^{32}$ suggesting that hAIP cells differentiated into enterocytes capable of transporting ions and water from apical to basolateral membrane. The hAIP cell cultures included cells expressing the enteroendocrine cell markers serotonin, chromogranin A, GIP, and synaptophysin; the Paneth cell marker lysozyme; and mucin-producing PASpositive cells (Figure 7a). By RT-PCR analysis of hAIP cell culture, we could detect not only several markers for enteroendocrine cells, but also the enterocyte markers Lfabp and sucrase-isomaltase, the goblet cell marker trefoil factor-3, and the Paneth cell marker lysozyme (Figure 7b). These data imply that hAIP cells have the potential to differentiate into four distinct types of intestinal epithelial cells. In contrast to the case of mouse FIP and AIP cell cultures, however, the removal of EGF from the culture medium seemed not to be required for differentiation of hAIP cells (Figure $7 \mathrm{~b}$ and Supplementary Figure 8). On the other hand, proliferation and survival of hAIP cells depended on the activation of both the PI3K/Akt cascade and the MAP kinase cascade in EGF signaling pathway, similar to those of mouse FIP and AIP cells (Figures $7 \mathrm{c}$ and $\mathrm{d}$ ). Moreover, hAIP cells preferred typeIV collagen-coated culture dishes for efficient adhesion and proliferation, whereas it was dispensable for mouse FIP and AIP cells (Figure 7c). Taken together, these results demonstrate that hAIP cells mainly composed of undifferentiated epithelial cells can be maintained in culture with continuous proliferation, and spontaneously give rise to differentiated epithelial cells in the presence of EGF and type-IV collagen.

\section{DISCUSSION}

The stem cell niche of the intestine is indispensable for the maintenance of undifferentiated epithelial cells through the life of organisms. The structure of the ISC niche seems simple, but the regulatory mechanism is complicated and strictly controlled by cross-talk between multiple signal pathways. ${ }^{3}$ An unbalanced regulation of these interactive signaling pathways accelerates proliferation of undifferentiated intestinal epithelial cells, and leads to polyp formation and subsequent tumorigenesis. In this study, we showed EGF as one of positive regulators for proliferation and survival of undifferentiated epithelial cells in the developing and adult mouse intestines. The epithelial-mesenchymal interaction between EGFR-expressing primitive intestinal epithelial cells and EGF-expressing adjacent fibroblasts in both the developing mouse epithelia and the crypts of adult mouse intestine is essential for activating proliferation and inhibiting apoptosis of ISCs and progenitor cells, implying the importance of EGF signaling for the maintenance of the ISC niche. We also demonstrate that, as well as in vivo, EGF promotes proliferation and decreases apoptosis in cultured immature cells, not only those isolated from developing mouse intestines, but also those from adult mouse and human intestines. Supplementation of the culture medium with EGF allows us to maintain for $>6$ months undifferentiated 

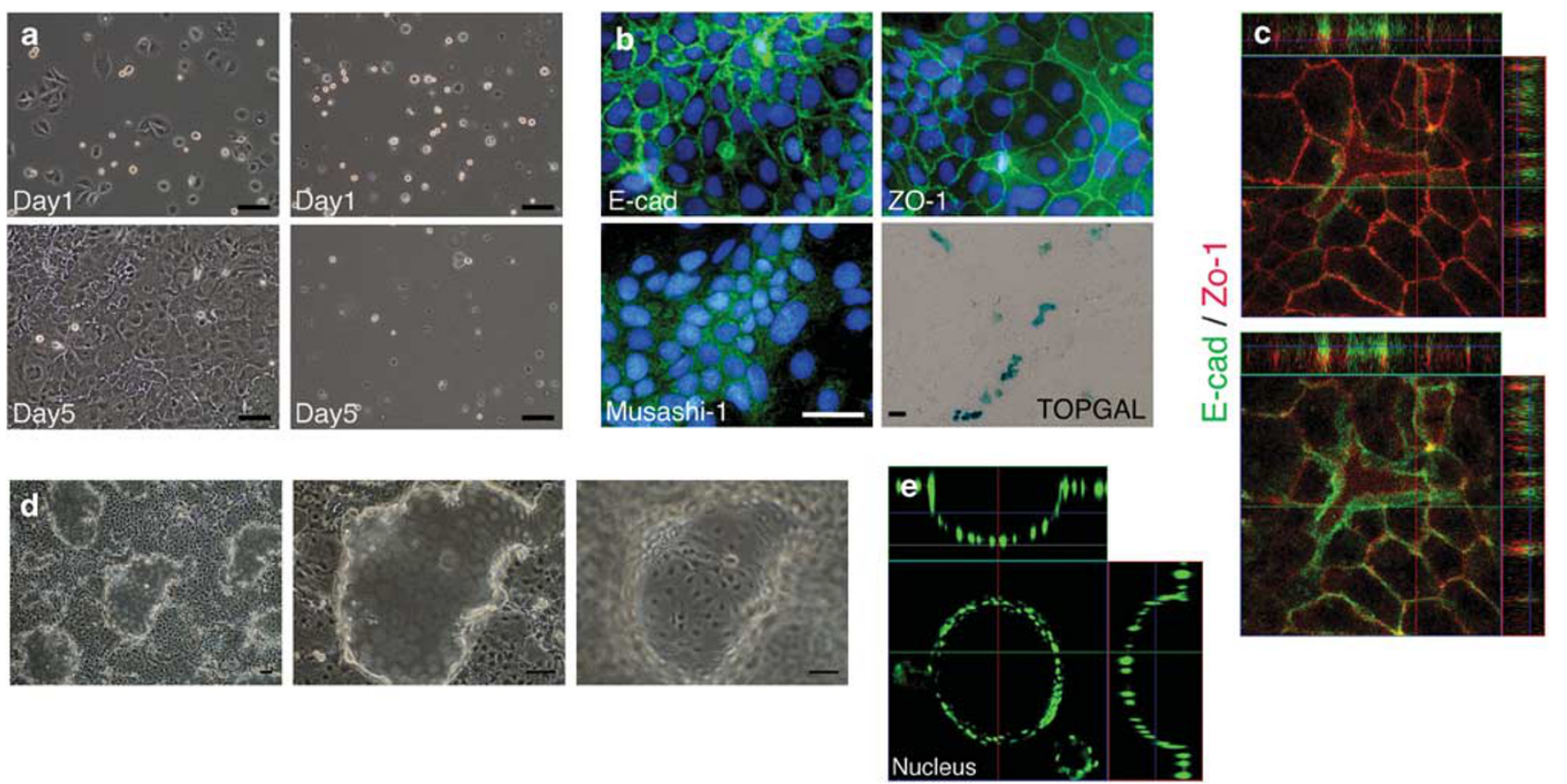

Figure 6 Long-term culture of hAIP cells. (a) Phase pictures of adult human intestinal cells cultured in SCM (left panels) or CS-2.0 medium (right panels) for 1 or 5 days on type-IV collagen-coated dishes, following the initial passage of cells. (b) Immunofluorescence staining of E-cad, Zo-1, and Musashi-1 for hAIP cells cultured in the presence of EGF. DNA is stained with DAPI. X-gal staining was also conducted for hAIP cells transfected with the TOP-GAL construct. (c) Confocal microscopic Z-stack analysis of hAIP cells stained with anti-Zo-1 (red) and anti-E-cad (green) antibodies. The upper and lower panels show images of the upper and lower parts of cells, respectively. (d) Phase pictures of dome-like structures formed in culture of hAIP cells. The left and middle or the right panel is in focus to the monolayer sheet of cells or an upper region of a dome-like structure, respectively. (e) Confocal microscopic Z-stack analysis of a dome-like structure formed from hAIP cells. The nuclei of cells were stained with SYTO13 as shown in green. Scale bar, $50 \mu \mathrm{m}$ (b) and $100 \mu \mathrm{m}(\mathbf{a}, \mathbf{d})$.

intestinal epithelial cells capable of giving rise to four distinct types of intestinal epithelial cells. In these cultured mouse FIP/AIP cells and hAIP cells, the PI3K/Akt cascade and the MAP kinase cascade are activated to transmit the EGF signal into the nuclei of these cells. PI3K/Akt signaling activates proliferation of ISCs and progenitor cells by closely linking to not only canonical Wnt signaling, through phosphorylation of $\beta$-catenin by activated Akt, but also BMP signaling through positive or negative regulation of PTEN activity by BMP or Noggin, respectively. ${ }^{12,13}$ Taken together with this evidence, our present data suggest that activation of PI3K/ Akt signaling in undifferentiated epithelial cells residing in the embryonic and the adult intestines depends on the presence of EGF, which stimulates proliferation and inhibits apoptosis of these cells directly or indirectly by interacting with other signaling pathways. In addition to EGF, however, additional factors that activate PI3K/Akt signaling may also contribute to proliferation of undifferentiated epithelial cells, because the number of $\mathrm{BrdU}^{+}$cells in culture of FIP and AIP cells with LY294002 was less than in culture without EGF. Previous reports demonstrated that continual activation of Akt in the PTEN-deficient ISCs is sufficient for polyp formation, and the inhibition of EGFR kinase activity can reduce the number of polyps, ${ }^{13,26}$ suggesting that EGF signaling is strictly controlled in order to balance the proliferation of undifferentiated epithelial cell in the maintenance of intestinal tissue homeostasis.

Owing to their finite lifetime, differentiated epithelial cells disappear from culture; therefore, intestinal epithelial cell culture requires the maintenance of viable ISCs and/or progenitor cells. In our culture system presented here, cells marked with Musashi-1 expression and $\beta$-catenin transcriptional activity (possible features of ISCs and progenitor cells), could be maintained with proliferation during a prolonged culture period and may contribute to the production of differentiated cells. In addition to these cells, however, a large number of cells that are not marked with Musashi-1 expression and $\beta$-catenin transcriptional activity present in the culture; these cells also proliferate extensively in response to EGF. In the crypts of adult mouse intestine, less than half of proliferating ISCs and progenitor cells consist of cells that express Musashi-1 and only a few cells are marked with $\beta$-catenin transcriptional activity. ${ }^{12,33}$ Thus, cells that proliferate in culture, but not express Musashi-1 and are not marked with $\beta$-catenin transcriptional activity, may be considered as another population of ISCs and progenitor cells. On the other hand, even in the presence of EGF, enterocyte marker Lfabp was detectable in cultures of mouse FIP/AIP cells, suggesting the coexistence of lineage committed, but still proliferating progenitors of enterocytes. In hAIP cell 
a
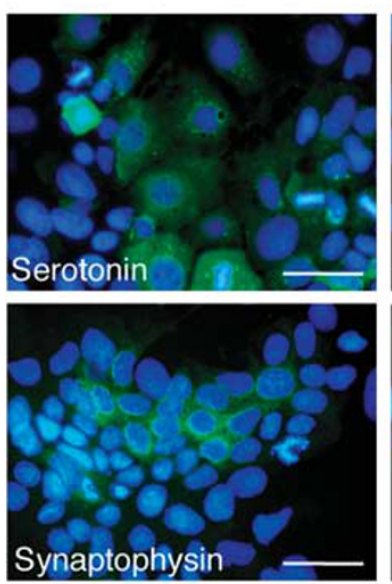
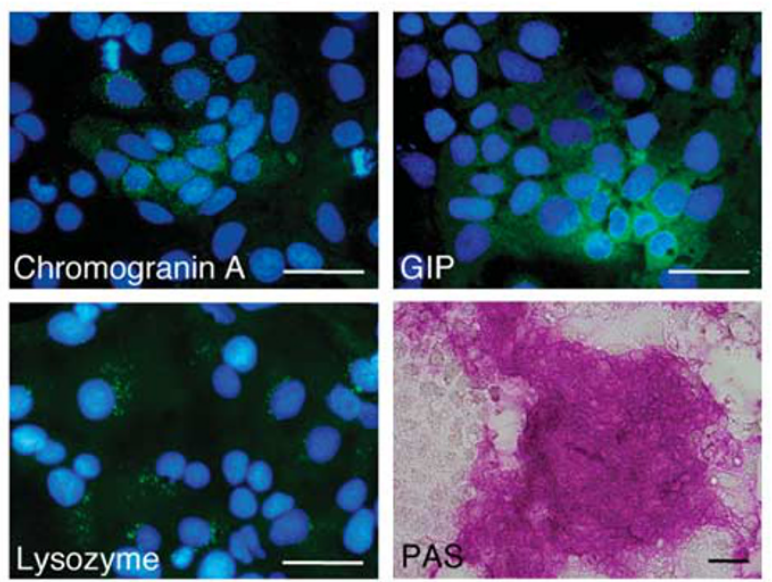

b

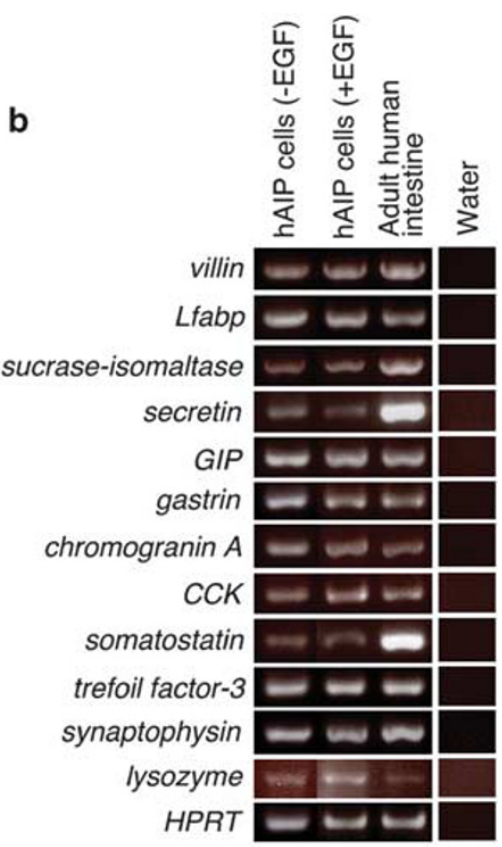

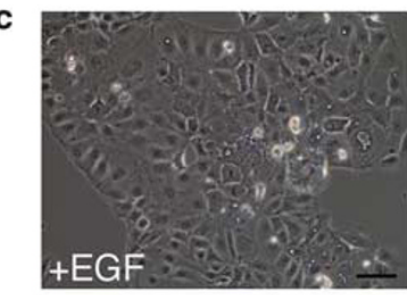

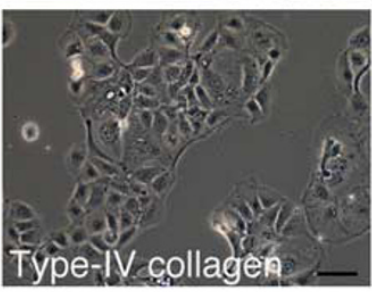

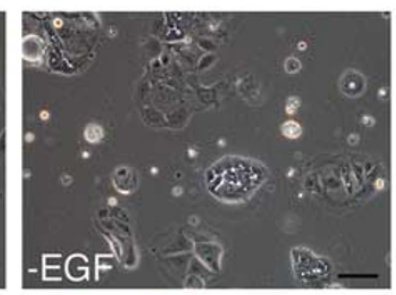

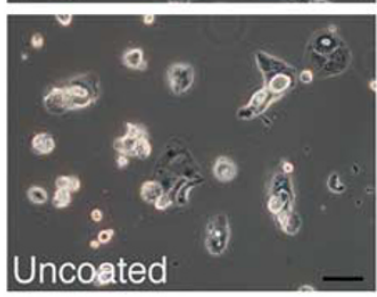

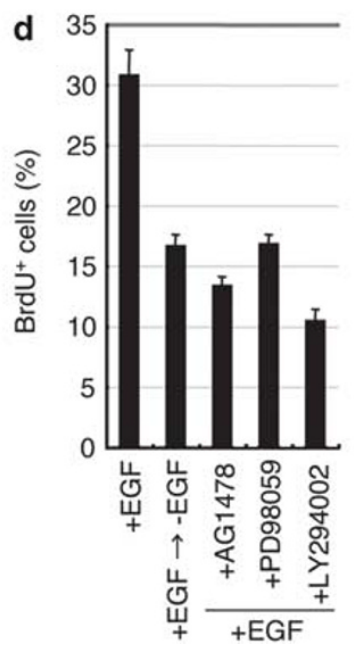

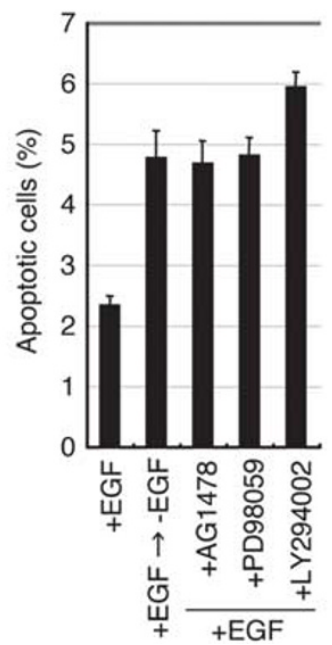

Figure 7 Characterization of hAIP cells in culture. (a) Immunofluorescence staining of serotonin, chromogranin A, GIP, synaptophysin, and lysozyme for hAIP cells cultured with EGF. DNA is stained with DAPI. PAS-positive cells were also found in culture of hAIP cells. (b) RT-PCR analysis of multiple intestinal gene expression in hAIP cells. These cells were cultured with EGF throughout the culture (shown as + EGF) or in the absence of EGF for 7 days after removing EGF from culture medium (shown as -EGF). As positive or negative controls, we used cDNA prepared from adult human intestines or water, respectively. (c) Phase pictures of hAIP cells cultured in the presence or absence of EGF for 4 days on type-IV collagen-coated dishes (upper panels), or cultured with EGF for 4 days on type-IV collagen-coated or -uncoated dishes (lower panels). (d) The percentages of BrdU ${ }^{+}$proliferating cells (left graph) and AnnexinV ${ }^{+}$apoptotic cells (right graph) were analyzed by flow cytometry. Before analyses, hAIP cells were cultured for 1 day with or without inhibitors for EGF signaling, such as AG1478, PD98059, or LY294002, in the presence of EGF. As control experiments, hAIP cells were also cultured with EGF throughout the culture, or in the absence of EGF for 1 day after removing EGF from culture medium. We supplemented the culture medium with inhibitors or removed EGF when cells occupied $70-80 \%$ of the surface of culture dishes. Scale bar, $50 \mu \mathrm{m}$ (a) and $100 \mu \mathrm{m}$ (c).

cultures, primitive intestinal cells are also maintained, but these cells spontaneously initiate differentiation; thus, four different types of intestinal epithelial cells co-exist in culture, regardless of whether EGF is present as a supplement to the culture medium. This evidence suggests that differentiation of intestinal epithelial cells is, at least partially, regulated by EGF, although this effect may differ between species and could be compensated by other signaling pathways.
Mouse FIP/AIP cells and hAIP cells maintain epithelial cell morphology, proliferate in response to EGF, and differentiate into four distinct types of intestinal epithelial cells in culture. There is no striking difference in these specific cellular characteristics among these three types of cells. Thus, the cell of origin of these cells is thought to be the same type of cells, such as ISCs and/or progenitor cells in the intestine. As mouse FIP/AIP cells and hAIP cells were retrospectively 
defined as undifferentiated intestinal epithelial cells, however, it cannot be ruled out that they would be derived from unidentified other lineage cells that could dedifferentiate or transdifferentiate into FIP and AIP cells in culture. Prospective isolation and clonal analysis of ISCs and progenitor cells will clarify whether isolated cells give rise to FIP and AIP cells.

Our present culture method allows differentiation of undifferentiated epithelial cells, but it is still difficult to uniform these cells into a particular cell population. For example, as a material for the screening of drugs, the absorptive enterocytes is expected to differentiate from cultured ISCs and/or progenitor cells. Thus, the intrinsic signal pathways that control differentiation of cultured mouse FIP/AIP cells and hAIP cells should be determined and modulated to induce differentiation of a specific, desired type of intestinal epithelial cells. Moreover, in order to mimic the function of differentiated intestinal epithelial cells in culture, the well-defined cellular architecture of epithelial cells should be reproduced. Although monolayer cell culture facilitates the maintenance and the analysis of cultured cells, the morphological features and polarization of these cells are often lost. Indeed, an electron microscopic analysis showed that mouse FIP/AIP cells have a flattened shape, implying a difference from the columnar-shaped normal epithelial cells in the intestine (data not shown). In contrast, hAIP cells spontaneously formed dome-like structures in which cells may mimic polarized enterocytes capable of transporting ions and water from apical to basolateral membrane, while the remaining cells in the monolayer sheet were still flat. The flat morphology of mouse FIP/AIP cells and hAIP cells differs from that of Caco-2 and other colon cancer cell lines, which can form polarized monolayers in culture..$^{20,32}$ Therefore, the morphological maturation of cultured normal intestinal epithelial cells will require further improvements in culture conditions, including a supply of an ideal set of ECM components and additional humoral factors. Normal intestinal epithelial cells that can reproduce in culture the gene expression pattern and well-defined morphological features of epithelial cells will be useful in developing therapeutic approaches, including repair of intestinal mucosa, and in screening the pharmacological effects of new compounds.

Supplementary Information accompanies the paper on the Laboratory Investigation website (http://www.laboratoryinvestigation.org)

\section{ACKNOWLEDGEMENTS}

We thank Elaine Fuchs for sharing a reagent, and Keiko Sueyoshi and Makiko Onishi for excellent technical assistance. This work was supported by grants from the Leading Project in Japan; the Program for Improvement of Research Environment for Young Researchers from Special Coordination Funds for Promoting Science and Technology commissioned by the Ministry of Education, Culture, Sports, Science and Technology of Japan; Takeda Science Foundation; and The Kao Foundation for Arts and Sciences.

Author contributions: Atsushi Suzuki: conception and design, collection and/or assembly of data, data analysis and interpretation, paper writing;
Sayaka Sekiya: collection and/or assembly of data; Eriko Gunshima: collection and/or assembly of data; Setsuko Fujii: collection and/or assembly of data; Hideki Taniguchi: conception and design, data analysis and interpretation, and final approval of the paper.

\section{DISCLOSURE/CONFLICT OF INTEREST}

The authors declare no conflict of interest.

1. Sancho $E$, Batlle $E$, Clevers $H$. Signaling pathways in intestinal development and cancer. Annu Rev Cell Dev Biol 2004:20:695-723.

2. Moore KA, Lemischka IR. Stem cells and their niches. Science 2006; 311:1880-1885.

3. Crosnier C, Stamataki D, Lewis J. Organizing cell renewal in the intestine: stem cells, signals and combinatorial control. Nat Rev Genet 2006;7:349-359.

4. Dauca M, Bouziges F, Colin S, et al. Development of the vertebrate small intestine and mechanisms of cell differentiation. Int J Dev Biol 1990;34:205-218.

5. Beaulieu JF. Differential expression of the VLA family of integrins along the crypt-villus axis in the human small intestine. J Cell Sci 1992;102: 427-436.

6. Groos S, Hunefeld G, Luciano L. Epithelial cell turnover-extracellular matrix relationship in the small intestine of human adults. Ital J Anat Embryol 2001;106:353-361.

7. Pinto $D$, Gregorieff $A$, Begthel $H$, et al. Canonical Wnt signals are essential for homeostasis of the intestinal epithelium. Genes Dev 2003; 17:1709-1713.

8. van Es JH, Jay $P$, Gregorieff $A$, et al. Wht signalling induces maturation of Paneth cells in intestinal crypts. Nat Cell Biol 2005;7:381-386.

9. Batlle $\mathrm{E}$, Henderson JT, Beghtel $\mathrm{H}$, et al. $\beta$-catenin and TCF mediate cell positioning in the intestinal epithelium by controlling the expression of EphB/ephrinB. Cell 2002;111:251-263.

10. Holmberg J, Genander M, Halford MM, et al. EphB receptors coordinate migration and proliferation in the intestinal stem cell niche. Cell 2006; 125:1151-1163.

11. Haramis AP, Begthel $H$, van den Born $M$, et al. De novo crypt formation and juvenile polyposis on BMP inhibition in mouse intestine. Science 2004;303:1684-1686.

12. He XC, Zhang J, Tong WG, et al. BMP signaling inhibits intestinal stem cell self-renewal through suppression of Wnt-beta-catenin signaling. Nat Genet 2004;36:1117-1121.

13. He XC, Yin T, Grindley JC, et al. PTEN-deficient intestinal stem cells initiate intestinal polyposis. Nat Genet 2007;39:189-198.

14. Morin PJ, Sparks $A B$, Korinek $V$, et al. Activation of $\beta$-catenin-Tcf signaling in colon cancer by mutations in $\beta$-catenin or APC. Science 1997;275:1787-1790.

15. Howe JR, Bair JL, Sayed MG, et al. Germline mutations of the gene encoding bone morphogenetic protein receptor $1 \mathrm{~A}$ in juvenile polyposis. Nat Genet 2001;28:184-187.

16. Howe JR, Roth S, Ringold JC, et al. Mutations in the SMAD4/DPC4 gene in juvenile polyposis. Science 1998;280:1086-1088.

17. Liaw D, Marsh DJ, Li J, et al. Germline mutations of the PTEN gene in Cowden disease, an inherited breast and thyroid cancer syndrome. Nat Genet 1997;16:64-67.

18. Fre $S$, Huyghe $M$, Mourikis $P$, et al. Notch signals control the fate of immature progenitor cells in the intestine. Nature 2005;435:964-968.

19. van $\mathrm{Es} \mathrm{JH}$, van Gijn ME, Riccio $\mathrm{O}$, et al. Notch/ $\gamma$-secretase inhibition turns proliferative cells in intestinal crypts and adenomas into goblet cells. Nature 2005:435:959-963.

20. Simon-Assmann $P$, Turck N, Sidhoum-Jenny $M$, et al. In vitro models of intestinal epithelial cell differentiation. Cell Biol Toxicol 2007;23: 241-256.

21. Quaroni A, Wands J, Trelstad RL, et al. Epithelioid cell cultures from rat small intestine. Characterization by morphologic and immunologic criteria. J Cell Biol 1979;80:248-265.

22. Perreault $\mathrm{N}$, Jean-Francois B. Use of the dissociating enzyme thermolysin to generate viable human normal intestinal epithelial cell cultures. Exp Cell Res 1996;224:354-364.

23. Simo P, Simon-Assmann P, Arnold C, et al. Mesenchyme-mediated effect of dexamethasone on laminin in cocultures of embryonic gut epithelial cells and mesenchyme-derived cells. J Cell Sci 1992;101:161-171. 
24. Kedinger M, Simon-Assmann P, Haffen K. Growth and differentiation of intestinal endodermal cells in a coculture system. Gut 1987;28: 237-241.

25. Abud HE, Watson N, Heath JK. Growth of intestinal epithelium in organ culture is dependent on EGF signalling. Exp Cell Res 2005; 303:252-262.

26. Torrance CJ, Jackson PE, Montgomery E, et al. Combinatorial chemoprevention of intestinal neoplasia. Nat Med 2000;6:1024-1028.

27. Suzuki A, Zheng YW, Kondo R, et al. Flow cytometric separation and enrichment of hepatic progenitor cells in the developing mouse liver. Hepatology 2000;32:1230-1239.

28. Suzuki A, Iwama A, Miyashita $\mathrm{H}$, et al. Role for growth factors and extracellular matrix in controlling differentiation of prospectively isolated hepatic stem cells. Development 2003;130:2513-2524.
29. Suzuki A, Nakauchi H, Taniguchi H. Glucagon-like peptide 1 [1-37] converts intestinal epithelial cells into insulin-producing cells. Proc Natl Acad Sci USA 2003;100:5034-5039.

30. Suzuki A, Zheng YW, Kaneko $S$, et al. Clonal identification and characterization of self-renewing pluripotent stem cells in the developing liver. J Cell Biol 2002;156:173-184.

31. Barker $\mathrm{N}$, van Es $\mathrm{JH}$, Kuipers J, et al. Identification of stem cells in small intestine and colon by marker gene Lgr5. Nature 2007;449:1003-1007.

32. Grasset $E$, Pinto $M$, Dussaulx $E$, et al. Epithelial properties of human colonic carcinoma cell line Caco-2: electrical parameters. Am J Physiol 1984;247:C260-C267.

33. Kayahara T, Sawada M, Takaishi S, et al. Candidate markers for stem and early progenitor cells, Musashi-1 and Hes1, are expressed in crypt base columnar cells of mouse small intestine. FEBS Lett 2003;535:131-135. 